\title{
Effects of Beetroot Juice Ingestion on Physical Performance in Highly Competitive Tennis Players
}

\author{
Álvaro López-Samanes $1, * \mathbb{(}$, Alberto Pérez-López ${ }^{2}\left(\mathbb{D}\right.$, Victor Moreno-Pérez ${ }^{3}$, \\ Fabio Yuzo Nakamura ${ }^{4}\left(\mathbb{D}\right.$, Jorge Acebes-Sánchez ${ }^{5}$ (D), Iñaki Quintana-Milla ${ }^{5}$, \\ Antonio J. Sánchez-Oliver ${ }^{6}{ }^{\mathbb{D}}$, Diego Moreno-Pérez ${ }^{7}$, Valentín Emilio Fernández-Elías ${ }^{8} \mathbb{D}$ and \\ Raúl Domínguez ${ }^{9}$ (i) \\ 1 School of Physiotherapy, Faculty of Health Sciences, Universidad Francisco de Vitoria, 28223 Madrid, Spain \\ 2 Department of Biomedical Sciences, Area of Sport and Physical Education, Faculty of Medicine and Health \\ Sciences, University of Alcalá, 28805 Madrid, Spain; alberto_perez-lopez@hotmail.com \\ 3 Center for Translational Research in Physiotherapy, Department of Pathology and Surgery, \\ Universidad Miguel Hernández, Elche, San Juan, 03202 Alicante, Spain; vmoreno@goumh.es \\ 4 Associate Graduate Program in Physical Education UPE/UFPB, 58051-970 João Pessoa, PB, Brazil; \\ fabioy_nakamura@yahoo.com.br \\ 5 Exercise and Sport Sciences, Faculty of Health Sciences, Universidad Francisco de Vitoria, 28223 Madrid, \\ Spain; j.acebes.prof@ufv.es (J.A.-S.); i.quintana.prof@ufv.es (I.Q.-M.) \\ 6 Departamento de Motricidad Humana y Rendimiento Deportivo, Universidad de Sevilla, 41013 Sevilla, \\ Spain; sanchezoliver@us.es \\ 7 Department of Education, Research and Evaluation Methods, Universidad Pontifica de Comillas, \\ 28015 Madrid, Spain; dmperez@comillas.edu \\ 8 Faculty of Sports Sciences, Universidad Europea de Madrid, 28670 Madrid, Spain; \\ valentin.fernandez@universidadeuropea.es \\ 9 College of Health Sciences, Isabel I University, 09003 Burgos, Spain; raul_dominguez_herrera@hotmail.com \\ * Correspondence: alvaro.lopez@ufv.es; Tel.: +34-91-709-1400 (ext. 1955)
}

Received: 9 September 2019; Accepted: 19 February 2020; Published: 23 February 2020

\begin{abstract}
Beetroot juice (BJ) contains high levels of inorganic nitrate $\left(\mathrm{NO}_{3}{ }^{-}\right)$and its intake has good evidence in increasing blood nitrate/nitrite concentrations. The ingestion of $\mathrm{BJ}$ has been associated with improvements in physical performance of endurance sports, however the literature in intermittent sports is scarce. The aim of this study was to investigate whether BJ could improve physical performance in tennis players. Thirteen well-trained tennis players ( $25.4 \pm 5.1$ years) participated in the study during their preparatory period for the tennis season. Subjects were randomly divided into two groups and performed a neuromuscular test battery after either BJ or placebo (PLA) consumption. Both trials were executed on two separate days, in randomized order, with one week of wash out period. The test battery consisted of serve velocity test (SVT), countermovement jump (CMJ), isometric handgrip strength (IHS), 5-0-5 agility test (5-0-5), and $10 \mathrm{~m}$ sprint (10-m). No significant differences were found in SVT $(1.19 \% ; p=0.536)$, CMJ $(0.96 \% ; p=0.327)$, IHS $(4.06 \% ; p=0.069), 5-0-5$ dominant and nondominant side $(1.11-2.02 \% ; p=0.071-0.191)$ and $10-\mathrm{m}(1.05 \% ; p=0.277)$ when comparing BJ and PLA ingestion. Thus, our data suggest that low doses of BJ (70 mL) consumption do not enhance tennis physical performance.
\end{abstract}

Keywords: NO precursors; racket sports; intermittent sports; ergogenic aid

\section{Introduction}

Tennis is an intermittent sport characterized by high-intensity efforts, such as accelerations, decelerations, running sprints, and frequent changes-of-direction (COD) [1], interspersed with periods 
of low-to-moderate intensity or rest (short breaks between points (10-20 s) and longer rest periods

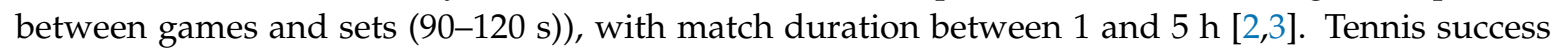
has been related to a mixture of technical, tactical, psychological, and physical components [4]. High performance in tennis is associated with higher values of strength and power output [5], agility [6], and serve velocity [7]. Other secondary determinant aspects have been proposed, such as environmental [8], nutrition [9], chronobiological aspects [10], and recovery strategies [11].

Due to the explosive demands that characterize tennis competition, identification of nutritional supplements and ergogenic aids that might contribute to enhance physical performance during training/matches could be an useful strategy to be adopted by elite tennis players [12]. However, despite the popularity of tennis around the world, studies developed on the effects of nutritional supplements with the objective of acutely enhancing tennis performance are scarce, reduced to a few studies on some ergogenic aids such as caffeine [13,14], creatine [15,16], sodium bicarbonate [17], or sodium citrate [18].

Beetroot juice (BJ), rich in inorganic nitrate $\left(\mathrm{NO}_{3}{ }^{-}\right)$, is considered to be an ergogenic aid as it may serve as a precursor of nitric oxide (NO) through the nitrate-nitrite-NO pathway [19]. Briefly, although $\mathrm{NO}_{3}{ }^{-}$is considered biologically inert, after $\mathrm{BJ}$ ingestion this molecule is reduced to nitrite $\left(\mathrm{NO}_{2}{ }^{-}\right)$ and subsequently to $\mathrm{NO}$ [20]. After absorption in the oral cavity or the intestine, circulating $\mathrm{NO}_{2}{ }^{-}$ serves as a substrate for $\mathrm{O}_{2}{ }^{-}$independent of $\mathrm{NO}$ generation, a process potentiated by hypoxia and acidosis conditions [20]. An increased $\mathrm{NO}$ presence derived from $\mathrm{NO}_{3}{ }^{-}$supplementation improves vasodilation, increasing blood flow to the muscles [21-23], and facilitates force production in vivo, reducing ATP cost in exercised muscles [24,25]. Hence, BJ supplementation has showed the capacity to improve endurance performance [26] and accelerate post-repeated-sprint recovery [27]. Furthermore, it has been suggested that ingestion of BJ may be particularly effective in intermittent sports at augmenting different physiological processes, such as calcium-handling proteins and contractile force in type II (fast-twitch) muscle fibers [28]. However, controversial findings are reported in the scientific literature, with some studies showing improvements in repeated-sprint performance by reducing time to reach peak power $(\sim 2.8)[29,30]$, while other studies do not report any difference between BJ and placebo (PLA) conditions [31]. In tennis, only one study developed by Aksit et al. [32] examined the relationships between simulated tennis performance test and circulating $\mathrm{NOx}$ (i.e., sum $\mathrm{NO}_{3}{ }^{-}+\mathrm{NO}_{2}{ }^{-}$) levels. However, no study has evaluated the effect of BJ ingestion in tennis. Thus, the aim of this study was to analyze the effect of $\mathrm{BJ}$ ingestion on physical tennis performance.

\section{Materials and Methods}

\subsection{Subjects}

Thirteen highly competitive male tennis players (age, $25.4 \pm 5.1$ years; body mass, $74.7 \pm 8.8 \mathrm{~kg}$; height, $1.82 \pm 0.1 \mathrm{~m}$; body mass index $(\mathrm{BMI}), 22.6 \pm 1.4$; tennis experience, $14.9 \pm 7.4$ years; hours training/week, $12.2 \pm 3.1 \mathrm{~h} /$ week) were recruited to participate in the study. Four tennis players had an ATP ranking (i.e., professional tennis ranking) between 650 and 1800, and nine were among the 350 best Spanish senior tennis players. After being fully informed of the experimental protocols, participants gave their informed written consent to participate. The Bioethics Commission of the University (number 46/2018) approved the study, which complied with the recommendations of the Declaration of Helsinki.

\subsection{Experimental Design}

The study design was randomized cross-over, placebo-controlled, and double-blind. Participants arrived at the training facilities on two separate days under the same experimental conditions with 1 week between protocols to allow recovery and substance wash out. Participants were instructed to avoid any form of exercise leading up to each test and the ingestion of caffeine/alcohol in the $24 \mathrm{~h}$ prior to the testing. In session 1, participants were subjected to a preliminary assessment of 
body composition and underwent a familiarization session of the neuromuscular test battery. Then, on two separate occasions (sessions 2 and 3), when they arrived at the tennis training facilities, participants were provided with a supplement containing $70 \mathrm{~mL}$ of either BJ (Beet IT; James White Drinks Ltd., Ipswich, UK) or PLA (ECO Saludviva, Alicante, Spain). The trial was double-blinded such that one researcher (D.M.-L.) allocated all the participants' drinks in a counter-balanced fashion (in each trial, 50\% of participants ingested PLA and 50\% ingested BJ beverages) with random assignment to each supplement (Research Randomizer, www.randomizer.org). Three hours after intake of BJ or PLA, subjects performed a standardized dynamic warm up protocol [33], and then a neuromuscular test battery was carried out, consisting of a tennis serve velocity test (SVT), countermovement jump (CMJ), isometric handgrip strength (IHS), 5-0-5 agility test (5-0-5), and $10 \mathrm{~m}$ sprint (10-m) (Figure 1). Furthermore, in both trials, the experimental procedures were performed at the same hour of the day to avoid the influence of circadian rhythms on performance [10].

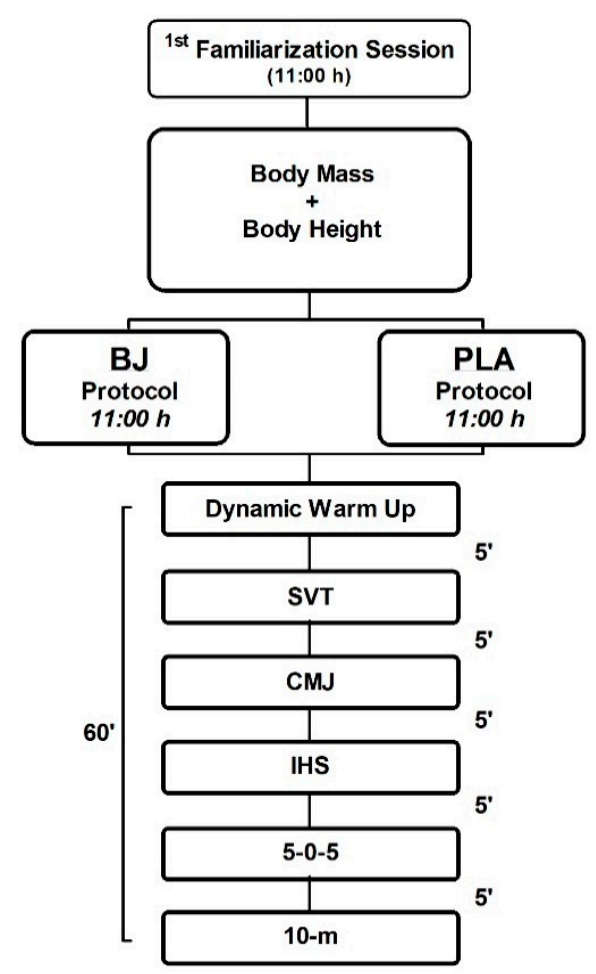

Figure 1. Experimental design flow chart. Abbreviations: BJ: beetroot juice; PLA: placebo; SVT: Serve velocity test; CMJ: counter movement jump; IHS: isometric handgrip strength; 5-0-5: Agility Test: 5-0-5; 10-m: 10 m sprint.

\subsection{Beetroot Juice vs. Placebo Ingestion}

In addition, the required sample size was determined by statistic power calculation on the basis of previous studies [27]. The minimum number of participants required to detect an $8 \% \pm 6 \%$ difference in counter movement jump (CMJ) performance between two groups, with a power of 0.80 and two-tailed $\alpha$ level set at 0.05 , was estimated as seven per group using the sample size package G Power 3.1 (Kiel, Germany). After an overnight fast, participants arrived to the laboratory $3 \mathrm{~h}$ before undertaking the neuromuscular test battery. Upon arrival, they were provided with a drink containing either $70 \mathrm{~mL}$ of BJ (containing $6.4 \mathrm{mmol}$ of $\mathrm{NO}_{3}{ }^{-}$) (Beet-It-Pro Elite Shot, James White Drinks Ltd., Ipswich, UK) or PLA (0.04 mmol of $\mathrm{NO}_{3}{ }^{-}$), as described elsewhere [34]. All participants were instructed to follow a diet sheet the day before each trial that consisted of $60 \%$ carbohydrates, $30 \%$ fat, and $10 \%$ proteins. Dietary $\mathrm{NO}_{3}{ }^{-}$was limited by providing subjects with a list of $\mathrm{NO}_{3}{ }^{--}$rich foods (e.g., beetroot, celery, or spinach), which they should avoid in the $48 \mathrm{~h}$ before each trial. In addition, in the $24 \mathrm{~h}$ leading up to each test, subjects were encouraged to avoid brushing their teeth or using any oral antiseptic rinse, or 
ingesting gum, sweets, stimulants (e.g., caffeine), or alcohol that could alter the oral microbiota and interfere with $\mathrm{NO}_{3}{ }^{-}$reduction. At the end of both trials, participants were asked to guess the order of the trials to determine whether they have identified in which day they ingested BJ and PLA.

\subsection{Environmental Conditions and Rate of Perceived Exertion (RPE)}

During the whole duration of each testing session, air temperature and humidity were measured with a portable weather station (WMR 108, Mextech, India). Data were averaged to obtain the mean morning and afternoon temperature $\left({ }^{\circ} \mathrm{C}\right)$ and relative humidity $(\%)$. The perceptual training intensity for each subject during the intervention was registered using the rating of perceived exertion (RPE) within 30 min of the warm up termination.

\subsection{SVT}

Serve speed was measured by a radar gun (model Pocket Radar Ball Coach PR1000BC, Republic of South Korea), which was set on "continuous mode" to detect maximal ball speed (40 to $210 \mathrm{~km} / \mathrm{h}$ range). Calibration was performed according to the manufacturer's specifications prior to each test. The serve test procedure was conducted as previously described [35]. Briefly, the radar was positioned in the tennis court in the center of the baseline, $4 \mathrm{~m}$ behind the server, aligned with the approximate height of ball contact $(\sim 2.2 \mathrm{~m})$ and pointing down the center of the court. After a brief warm up consisting of dynamic movements of the shoulder, five-second services, and five submaximal services, the serve velocity test was performed. Participants were required to serve in a $1 \times 1 \mathrm{~m}^{2}$ area allocated in the farther diagonal corner of the serving area, performing five maximal speed serves in as few attempts as possible. The mean velocity of the five serves was calculated for further analysis.

\section{6. $\mathrm{CMJ}$}

Participants completed three repetitions of a CMJ using an infrared jump system (Optojump, Microgate, Italy) according to standard methodology [36]. Each participant performed three maximal CMJ interspersed with $45 \mathrm{~s}$ of passive recovery. The highest height out of the three jumps was recorded.

\subsection{IHS}

Two maximum isometric voluntary contractions were measured in the dominant hand using a calibrated handgrip dynamometer (Takei 5101, Tokyo, Japan). Volunteers sat with 0 degrees of shoulder flexion, 0 degrees of elbow flexion, and the forearm and hand in a neutral position [10]. The highest value out of two attempts was recorded as the maximum voluntary handgrip strength.

\section{8. $5-0-5$}

The athletes' ability to perform a single, rapid $180^{\circ}$ change of direction over a $5 \mathrm{~m}$ distance was measured (Smartspeed, Fusion Sport, Australia) using a modified version (stationary start) of the 5-0-5 agility test. Players started with their preferred foot behind the starting position (dominant (DOM) or nondominant side (NO-DOM)) and accelerated voluntarily, sprinting with maximal effort without a racquet [37]. Each repetition was initiated from a standing position, $50 \mathrm{~cm}$ behind the photocell gate which started a digital timer. Two trials were completed, one each pivoting the left and right feet, and the highest value out of the two attempts was recorded for subsequent analysis.

\section{9. $10-m$}

The time during a $10 \mathrm{~m}$ dash in a straight line was measured using two photocell gates placed $1 \mathrm{~m}$ above the ground level (Smartspeed, Fusion Sport, Australia). Each sprint was initiated from a standing position, $50 \mathrm{~cm}$ behind the photocell gate which started a digital timer. Another photocell gate was placed at the finish line to stop the timer [10]. The best performance out of two repetitions, separated by $1 \mathrm{~min}$ recovery period, was recorded for subsequent analysis. 


\subsection{Statistical Analysis}

Data are presented as means and standard deviation. The Shapiro-Wilk test was used to assess the distribution of data. All variables were compared using the Student's t-test for related variables (BJ vs. PLA). The significance level was set at $p \leq 0.05$. Cohen's $d$ formula for effect size (ES) was used, and the results were based on the following criteria: trivial (0-0.19), small (0.20-0.49), medium $(0.50-0.79)$, and large ( 0.80 and greater) [38]. All the statistical analyses were performed using SPSS software, version 22.0 (SPSS Inc., Chicago, IL, USA).

\section{Results}

\subsection{Enviromental Conditions and RPE}

Ambient condition data were averaged to obtain the mean morning temperature $\left(11.0 \pm 3.4^{\circ} \mathrm{C}\right.$, $p=0.383)$ and relative humidity $(36 \% \pm 5 \%, p=0.792)$. Regarding RPE values, no differences were founded between BJ and PLA conditions ( 3.8 vs. 4.2, $p=0.248$ ). In addition, the order of the experimental trials was correctly identified by $53.8 \%$ of the participants ( 7 of 13 ).

\subsection{SVT, CMJ, IHS, 10-m, and 5-0-5 Tests}

No significant differences were founded between conditions (BJ vs. PLA) in any of the physical performance tests analyzed, such as SVT $(p=0.536$; ES $=0.16$; Figure $2 \mathrm{a}), \mathrm{CMJ}$ height $(p=0.327$; $\mathrm{ES}=-0.14$; Figure $2 \mathrm{~b})$, IHS test $(p=0.069 ; \mathrm{ES}=0.26$; Figure $2 \mathrm{c}), 5-0-5 \mathrm{DOM}(p=0.071 ; \mathrm{ES}=0.69$; Figure 2d), 5-0-5 NO-DOM ( $p=0.191$; ES = -0.38; Figure 2e), and 10-m test $(p=0.277 ; \mathrm{ES}=0.39$; Figure 2f).
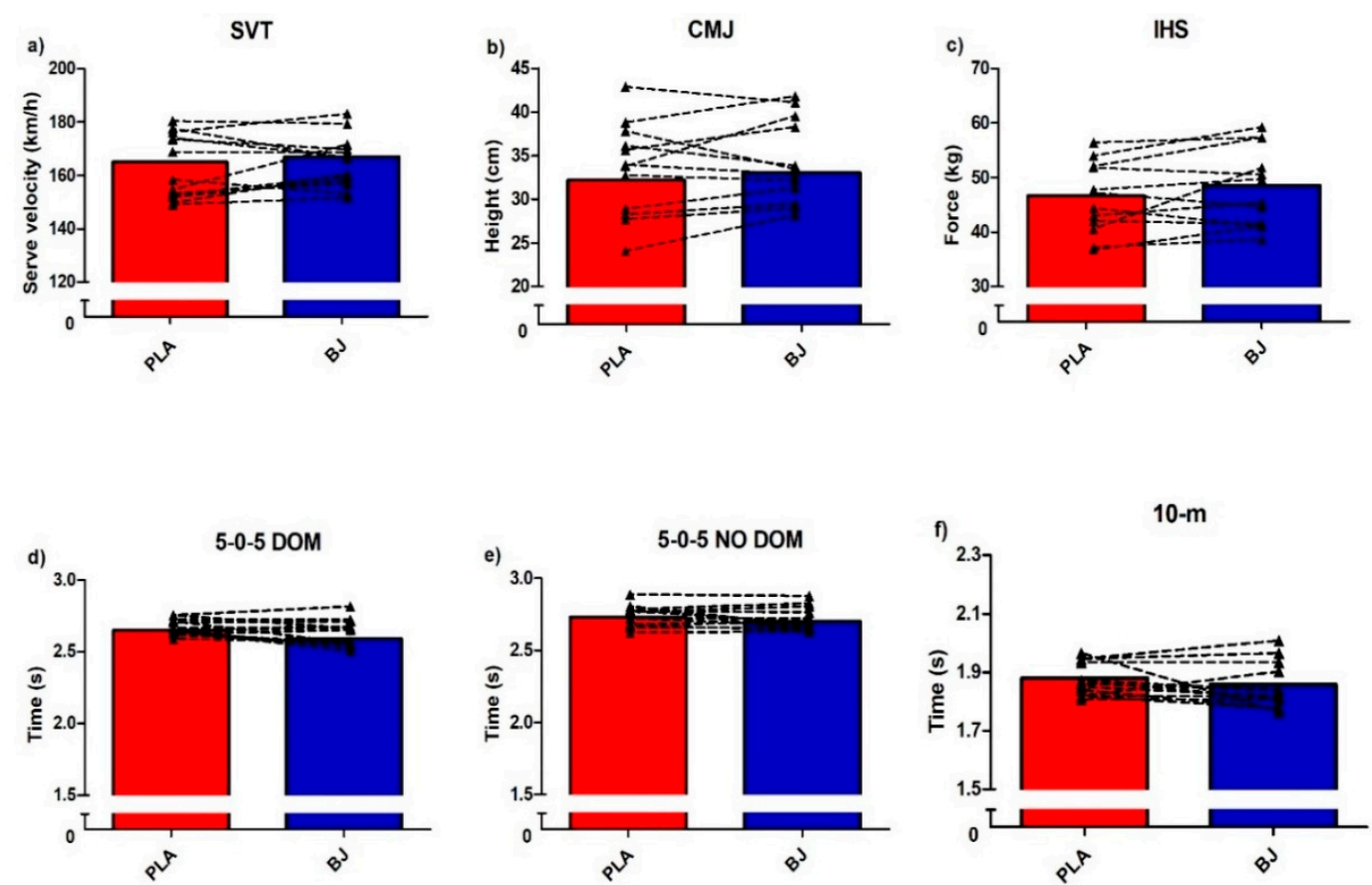

Figure 2. Effects of beetroot juice (BJ) or placebo (PLA) on (a) SVA: serve velocity test; (b) CMJ: countermovement vertical jump height; (c) IHS: isometric handgrip strength; (d) 5-0-5 DOM: agility test dominant side; (e) 5-0-5 NO-DOM: agility test nondominant side; (f) 10-m: $10 \mathrm{~m}$ sprint.

\section{Discussion}

The ergogenicity of BJ has been evidenced in some intermittent sports (e.g., soccer, rugby, hockey) [39-41], however, other sports such as basketball showed no benefits with BJ ingestion [42]. To our knowledge, no previous studies have evaluated its efficacy on tennis physical performance. 
Thus, there is a need to understand how BJ can impact physical performance of tennis players to ascertain if the ingestion of BJ might be beneficial. In comparison with PLA values, BJ ingestion showed no statistical differences in the performance variables analyzed, such as SVT (1.2\%), IHS (4.1\%), 10-m $(-1.1 \%)$ and 5-0-5 in the DOM $(-2.0 \%)$ and NO-DOM side $(-1.1 \%)$. Overall, the results indicate that $\mathrm{BJ}$ in a dose of $70 \mathrm{~mL}$ does not produce an improvement of physical tennis performance in highly trained tennis players, that is in agreement with previous studies in other intermittent team sports (e.g., basketball), where no significant differences were found in agility, sprint, isometric strength, or even match play demands values [42].

Beetroot juice consumption promotes an enhanced endurance performance in several populations of athletes [26], potentially due to improved vasodilatation, which facilitates an increase in blood flow to the exercised muscles [22,23] and stimulates a reduction in the $\mathrm{O}_{2}$ needed during submaximal efforts [25]. However, in intermittent efforts, there is controversial evidence regarding the ergogenic effect of BJ [40,42]. While several days of BJ administration may stimulate a modification of contractile properties of the muscle, allowing the generation of higher rates of power, enhancing speed in short distances and duration tests $[27,29,30]$, no significant differences were found after the acute ingestion of $\mathrm{BJ}[28,36]$. In this study, we observed no changes in the different explosive actions related to physical tennis performance (e.g., SVT, CMJ, HIS, 5-0-5, and 10-m) with the ingestion of BJ against PLA.

Therefore, in this study, the acute ingestion of low doses of $\mathrm{NO}_{3}{ }^{-}(6.4 \mathrm{mmol})$ did not stimulate an increase in physical performance in tennis. These results are opposed to the evidence provided by Coggan et al. [43], who found an increased muscle speed and power in healthy men and women after isokinetic knee extension performed at high velocity (360 degree/s), a task where type II muscle fibers are highly recruited and expected to generate elevated force and power production. However, the $\mathrm{NO}_{3}$ dose provided in both studies was substantially different (6.4 vs. $11.2 \mathrm{mmol}$ ) and, thus, low doses of $\mathrm{NO}_{3}$ may not be enough to cause an improvement of contractile properties of the muscle, potentially due to insufficient activation of cyclic guanosine monophosphate (cGMP) [43].

The tennis serve is a technical/tactical skill which plays a critical role in the outcome of a tennis match, and, particularly, ball velocity is a key variable in determining a successful play [44]. We observed that serve velocity increased $1.2 \%$ with BJ ingestion, not reaching statistical significance in our study $(p=0.536)$. Although no previous studies have been developed with the ingestion of BJ on physical tennis performance, the ingestion of other ergogenic aids or nutritional supplements such as caffeine (3.77\% improvement against PLA) in tennis serve [13] or sports drinks reducing fatigue after tennis match [45] have demonstrated higher effects in tennis performance compared with BJ ingestion. Also, vertical jumps are commonly involved during tennis training/matches in different tennis-specific actions (e.g., serves/smashes). According to previous literature, the ingestion of BJ in small doses $(70 \mathrm{~mL})$ does not improve jump height $(2.3 \%$ improvement without reaching statistical significance) [46]. These results are in agreement with the data obtained in our study $(p=0.327)$. The differences found in both studies could be attributed to the differences in the sample characteristics. In our study, we studied highly trained male tennis players, while the study of Cuenca et al. [46] was conducted with young active male players. In addition to the evidence previously reported, some studies did not find a positive effect of $\mathrm{NO}_{3}{ }^{-}$supplementation on peak power during a cycle ergometer test $[29,47]$, while other studies found improvement in peak power on a cycle ergometer $[46,48,49]$ and strength performance [50,51], but not during a vertical jump [46]. Hence, controversial evidence about the effects of BJ supplementation on several aspects of physical performance, such as muscle force or power, are presented in the literature.

In comparison with PLA, BJ ingestion did not stimulate an increased IHS on the dominant hand, $(4.1 \%, p=0.069)$. Our data are in agreement with a previous study published by Clifford et al. [25] which showed no statistical differences in maximal isometric voluntary contractions (1.86\%) after ingestion of BJ in different team sport athletes (e.g., soccer, rugby, etc.) Although IHS is not a specific action of tennis match play, this test constitutes a simple method to test the effect of BJ on force production and might be an indication of higher force capacity. In addition, Girard et al. [5] established 
that isometric handgrip strength values are related to higher physical performance in adolescent tennis players. However, the acute administration of BJ did not cause a significant effect on this test.

During tennis performance, the ability to change direction on the tennis court has been related to tennis performance since during a tennis point, players complete an average of four directional changes per point, reaching up to 15 directional changes during long rallies [52]. In fact, according to Roetert et al. [6], agility could be the only physical performance variable predictive of competitive rankings in young male tennis players. In our study, no statistically significant differences were found after BJ ingestion in the DOM $(-2.02 \%)$ and NO-DOM side $(-1.11 \%)$, compared with PLA ingestion agility values $(p=0.071)$. In conjunction with the ability of change-of-direction on the tennis court, the capacity to cover short distances (i.e., $<20 \mathrm{~m}$ ) in less time is also critical for tennis performance and has been related to higher tennis physical performance. According to previous literature, controversial findings have been reported regarding the ergogenic effect of BJ on sprint performance. While some investigations reported benefits from BJ ingestion (140 $\mathrm{mL})$ [40], other studies did not find an effect $[42,53]$. To our knowledge, this is the first study to analyze the effects of BJ on agility in tennis.

\section{Limitations}

BJ dose $(70 \mathrm{~mL})$ used during this study was insufficient to stimulate an ergogenic effect. While some studies have reported improvements with this dose in high-intensity efforts [34,46], one study found that a dose of $4.2 \mathrm{mmol}$ of $\mathrm{NO}_{3}{ }^{-}(70 \mathrm{~mL})$ was insufficient to improve the time to task failure; however the authors observed increments with doses between $8.4 \mathrm{mmol}$ of $\mathrm{NO}_{3}{ }^{-}(140 \mathrm{~mL})$ and $16.8 \mathrm{mmol}$ of $\mathrm{NO}_{3}{ }^{-}(280 \mathrm{~mL})$ [30]. Further studies in which a larger sample size will be recruited are required to corroborate the results obtained in this study $(n=13)$. In addition, other limitations of our study were that $\mathrm{NO}_{2}{ }^{-}$and $\mathrm{NO}_{3}{ }^{-}$plasma concentrations were not measured and the authors have limited ability to alter the diet in these athletes, potentially compromising their dietary restriction of nitrate-containing foods. Thus, despite the fact that our research reported no ergogenic effects after the ingestion of BJ, higher doses of BJ may promote an ergogenic effect, and, thus, future studies should examine the effects of higher doses of NO precursors than the one used in the current study.

\section{Conclusions}

In summary, the results of this study indicate that the ingestion of $70 \mathrm{~mL}$ of $\mathrm{BJ}(4.2 \mathrm{mmol})$ does not improve serve velocity, jump height, isometric handgrip strength, agility performance, and sprint speed in highly trained tennis players. Therefore, low doses of NO precursors may not stimulate ergogenic effects on tennis physical performance.

Author Contributions: Á.L.-S., V.M.-P., A.P-L and R.D. conceived and designed the experiments; Á.L.-S., V.M.-P., D.M.-P. and R.D. recruited the subjects; A.P.-L., A.J.S.-O., and R.D. elaborated and ensured that subjects followed the diet guidelines; A.J.S.-O. ensured the randomization; Á.L.-S., A.P.-L., V.M.-P., J.A.-S., I.Q.-M., D.M.-P., and V.E.F.-E performed the experiment; J.A.-S., I.Q.-M., D.M.-P., and V.E.F.-E extracted the data; Á.L.-S., A.P.-L. and V.E.F.-E. conducted the statistical analysis; J.A.-S., I.Q.-M. and D.M.-P. elaborated the figures; Á.L.-S., A.P.-L. and F.Y.N. wrote the original draft of the manuscript; Á.L.-S., V.M.-P., A.P-L., I.Q.-M., A.J.S.-O., D.M.-P., V.E.F.-E. and R.D. revised the manuscripts; All authors have read and agreed to the published version of the manuscript.

Funding: This study was partially funded by Universidad Francisco de Vitoria and Banco Santander via grants (UFV-2020/44).

Acknowledgments: We would like to thank the participants for their participation in this project.

Conflicts of Interest: No potential conflict of interest was reported by the authors. 


\section{References}

1. Lopez-Samanes, A.; Pallares, J.G.; Perez-Lopez, A.; Mora-Rodriguez, R.; Ortega, J.F. Hormonal and neuromuscular responses during a singles match in male professional tennis players. PLoS ONE 2018, 13, e0195242. [CrossRef] [PubMed]

2. Kovacs, M.S. Tennis physiology: Training the competitive athlete. Sports Med. 2007, 37, 189-198. [CrossRef] [PubMed]

3. Fernandez, J.; Mendez-Villanueva, A.; Pluim, B.M. Intensity of tennis match play. Br. J. Sports Med. 2006, 40, 387-391. [CrossRef] [PubMed]

4. Kovacs, M.S. Applied physiology of tennis performance. Br. J. Sports Med. 2006, 40, 381-385. [CrossRef] [PubMed]

5. Girard, O.; Millet, G.P. Physical determinants of tennis performance in competitive teenage players. J. Strength Cond. Res. 2009, 23, 1867-1872. [CrossRef]

6. Roetert, P.; Garrett, G.; Brown, S.; Camaione, D.N. Performance profiles of nationally ranked junior tennis players. J. Strength Cond. Res. 1992, 6, 225-231.

7. Ulbricht, A.; Fernandez-Fernandez, J.; Mendez-Villanueva, A.; Ferrauti, A. Impact of Fitness Characteristics on Tennis Performance in Elite Junior Tennis Players. J. Strength Cond. Res. 2016, 30, 989-998. [CrossRef]

8. Ellenbecker, T.S.; Stroia, K.A. Heat research guides current practices in professional tennis. Br. J. Sports Med. 2014, 48, 5-6. [CrossRef]

9. Lopez-Samanes, A.; Moreno-Perez, V.; Kovacs, M.S.; Pallares, J.G.; Mora-Rodriguez, R.; Ortega, J.F. Use of nutritional supplements and ergogenic aids in professional tennis players. Nutr. Hosp. 2017, 34, 1463-1468. [CrossRef]

10. Lopez-Samanes, A.; Moreno-Perez, D.; Mate-Munoz, J.L.; Dominguez, R.; Pallares, J.G.; Mora-Rodriguez, R.; Ortega, J.F. Circadian rhythm effect on physical tennis performance in trained male players. J. Sports Sci. 2017, 35, 2121-2128. [CrossRef]

11. Duffield, R.; Murphy, A.; Kellett, A.; Reid, M. Recovery from repeated on-court tennis sessions: Combining cold-water immersion, compression, and sleep recovery interventions. Int. J. Sports Physiol. Perform. 2014, 9, 273-282. [CrossRef] [PubMed]

12. Lopez-Samanes, A.; Ortega, J.F.; Fernandez-Elias, V.; Borreani, S.; Maté-Muñoz, J.; Kovacs, M.S. Nutritional Ergogenic Aids in Tennis: A Brief Review. Strength Cond. J. 2015, 37, 1-11. [CrossRef]

13. Hornery, D.J.; Farrow, D.; Mujika, I.; Young, W.B. Caffeine, carbohydrate, and cooling use during prolonged simulated tennis. Int. J. Sports Physiol. Perform. 2007, 2, 423-438. [CrossRef] [PubMed]

14. Gallo-Salazar, C.; Areces, F.; Abian-Vicen, J.; Lara, B.; Salinero, J.J.; Gonzalez-Millan, C.; Portillo, J.; Munoz, V.; Juarez, D.; Del Coso, J. Enhancing physical performance in elite junior tennis players with a caffeinated energy drink. Int. J. Sports Physiol. Perform. 2015, 10, 305-310. [CrossRef] [PubMed]

15. Pluim, B.M.; Ferrauti, A.; Broekhof, F.; Deutekom, M.; Gotzmann, A.; Kuipers, H.; Weber, K. The effects of creatine supplementation on selected factors of tennis specific training. Br. J. Sports Med. 2006, 40, 507-511, discussion 511-502. [CrossRef] [PubMed]

16. Eijnde, B.O.; Vergauwen, L.; Hespel, P. Creatine loading does not impact on stroke performance in tennis. Int. J. Sports Med. 2001, 22, 76-80. [CrossRef]

17. Wu, C.L.; Shih, M.C.; Yang, C.C.; Huang, M.H.; Chang, C.K. Sodium bicarbonate supplementation prevents skilled tennis performance decline after a simulated match. J. Int. Soc. Sports Nutr. 2010, 7, 33. [CrossRef]

18. Cunha, V.C.R.; Aoki, M.S.; Zourdos, M.C.; Gomes, R.V.; Barbosa, W.P.; Massa, M.; Moreira, A.; Capitani, C.D. Sodium citrate supplementation enhances tennis skill performance: A crossover, placebo-controlled, double blind study. J. Int. Soc. Sports Nutr. 2019, 16, 32. [CrossRef]

19. Dominguez, R.; Mate-Munoz, J.L.; Cuenca, E.; Garcia-Fernandez, P.; Mata-Ordonez, F.; Lozano-Estevan, M.C.; Veiga-Herreros, P.; da Silva, S.F.; Garnacho-Castano, M.V. Effects of beetroot juice supplementation on intermittent high-intensity exercise efforts. J. Int. Soc. Sports Nutr. 2018, 15, 2. [CrossRef]

20. Lundberg, J.O.; Weitzberg, E. NO-synthase independent NO generation in mammals. Biochem. Biophys. Res. Commun. 2010, 396, 39-45. [CrossRef]

21. Ferguson, S.K.; Holdsworth, C.T.; Wright, J.L.; Fees, A.J.; Allen, J.D.; Jones, A.M.; Musch, T.I.; Poole, D.C. Microvascular oxygen pressures in muscles comprised of different fiber types: Impact of dietary nitrate supplementation. Nitric Oxide Biol. Chem. 2015, 48, 38-43. [CrossRef] [PubMed] 
22. Walker, M.A.; Bailey, T.G.; McIlvenna, L.; Allen, J.D.; Green, D.J.; Askew, C.D. Acute Dietary Nitrate Supplementation Improves Flow Mediated Dilatation of the Superficial Femoral Artery in Healthy Older Males. Nutrients 2019, 11, 954. [CrossRef] [PubMed]

23. Shepherd, A.I.; Costello, J.T.; Bailey, S.J.; Bishop, N.; Wadley, A.J.; Young-Min, S.; Gilchrist, M.; Mayes, H.; White, D.; Gorczynski, P.; et al. "Beet" the cold: Beetroot juice supplementation improves peripheral blood flow, endothelial function, and anti-inflammatory status in individuals with Raynaud's phenomenon. J. Appl. Physiol. 2019, 127, 1478-1490. [CrossRef] [PubMed]

24. Haider, G.; Folland, J.P. Nitrate supplementation enhances the contractile properties of human skeletal muscle. Med. Sci. Sports Exerc. 2014, 46, 2234-2243. [CrossRef] [PubMed]

25. Bailey, S.J.; Fulford, J.; Vanhatalo, A.; Winyard, P.G.; Blackwell, J.R.; DiMenna, F.J.; Wilkerson, D.P.; Benjamin, N.; Jones, A.M. Dietary nitrate supplementation enhances muscle contractile efficiency during knee-extensor exercise in humans. J. Appl. Physiol. 2010, 109, 135-148. [CrossRef]

26. Dominguez, R.; Cuenca, E.; Mate-Munoz, J.L.; Garcia-Fernandez, P.; Serra-Paya, N.; Estevan, M.C.; Herreros, P.V.; Garnacho-Castano, M.V. Effects of Beetroot Juice Supplementation on Cardiorespiratory Endurance in Athletes. A Systematic Review. Nutrients 2017, 6, 43. [CrossRef]

27. Clifford, T.; Berntzen, B.; Davison, G.W.; West, D.J.; Howatson, G.; Stevenson, E.J. Effects of Beetroot Juice on Recovery of Muscle Function and Performance between Bouts of Repeated Sprint Exercise. Nutrients 2016, 8, 506. [CrossRef]

28. Jones, A.M.; Ferguson, S.K.; Bailey, S.J.; Vanhatalo, A.; Poole, D.C. Fiber Type-Specific Effects of Dietary Nitrate. Exerc. Sport Sci. Rev. 2016, 44,53-60. [CrossRef]

29. Jonvik, K.L.; Nyakayiru, J.; Van Dijk, J.W.; Maase, K.; Ballak, S.B.; Senden, J.M.G.; Van Loon, L.J.C.; Verdijk, L.B. Repeated-sprint performance and plasma responses following beetroot juice supplementation do not differ between recreational, competitive and elite sprint athletes. Eur. J. Sport Sci. 2018, 18, 524-533. [CrossRef]

30. Wylie, L.J.; Kelly, J.; Bailey, S.J.; Blackwell, J.R.; Skiba, P.F.; Winyard, P.G.; Jeukendrup, A.E.; Vanhatalo, A.; Jones, A.M. Beetroot juice and exercise: Pharmacodynamic and dose-response relationships. J. Appl. Physiol. 2013, 115, 325-336. [CrossRef]

31. Tatlici, A.; Çakmakci, O. The effects of acute dietary nitrate supplementation on anaerobic power of elite boxers. Med. Dello Sport 2019, 72, 225-233.

32. Aksit, T.; Turgay, F.; Kutlay, E.; M, Z.O.; Vural, F. The relationships between simulated tennis performance and biomarkers for nitric oxide synthesis. J. Sports Sci. Med. 2013, 12, 267-274. [PubMed]

33. Ayala, F.; Moreno-Perez, V.; Vera-Garcia, F.J.; Moya, M.; Sanz-Rivas, D.; Fernandez-Fernandez, J. Acute and Time-Course Effects of Traditional and Dynamic Warm-Up Routines in Young Elite Junior Tennis Players. PLoS ONE 2016, 11, e0152790. [CrossRef] [PubMed]

34. Dominguez, R.; Garnacho-Castano, M.V.; Cuenca, E.; Garcia-Fernandez, P.; Munoz-Gonzalez, A.; de Jesus, F.; Lozano-Estevan, M.D.C.; Fernandes da Silva, S.; Veiga-Herreros, P.; Mate-Munoz, J.L. Effects of Beetroot Juice Supplementation on a 30-s High-Intensity Inertial Cycle Ergometer Test. Nutrients 2017, 9, 1360. [CrossRef]

35. Moreno-Perez, V.; Lopez-Samanes, A.; Dominguez, R.; Fernandez-Elias, V.E.; Gonzalez-Frutos, P.; Fernandez-Ruiz, V.; Perez-Lopez, A.; Fernandez-Fernandez, J. Acute effects of a single tennis match on passive shoulder rotation range of motion, isometric strength and serve speed in professional tennis players. PLoS ONE 2019, 14, e0215015. [CrossRef]

36. Bosco, C.; Luhtanen, P.; Komi, P.V. A simple method for measurement of mechanical power in jumping. Eur. J. Appl. Physiol. Occup. Physiol. 1983, 50, 273-282. [CrossRef]

37. Gallo-Salazar, C.; Del Coso, J.; Barbado, D.; Lopez-Valenciano, A.; Santos-Rosa, F.J.; Sanz-Rivas, D.; Moya, M.; Fernandez-Fernandez, J. Impact of a competition with two consecutive matches in a day on physical performance in young tennis players. Appl. Physiol. Nutr. Metab. Physiol. Appl. Nutr. Metab. 2017, 42, 750-756. [CrossRef]

38. Cohen, J. A power primer. Psychol. Bull. 1992, 112, 155-159. [CrossRef]

39. Nyakayiru, J.; Jonvik, K.L.; Trommelen, J.; Pinckaers, P.J.; Senden, J.M.; van Loon, L.J.; Verdijk, L.B. Beetroot Juice Supplementation Improves High-Intensity Intermittent Type Exercise Performance in Trained Soccer Players. Nutrients 2017, 9, 314. [CrossRef]

40. Thompson, C.; Wylie, L.J.; Fulford, J.; Kelly, J.; Black, M.I.; McDonagh, S.T.; Jeukendrup, A.E.; Vanhatalo, A.; Jones, A.M. Dietary nitrate improves sprint performance and cognitive function during prolonged intermittent exercise. Eur. J. Appl. Physiol. 2015, 115, 1825-1834. [CrossRef] 
41. Thompson, C.; Vanhatalo, A.; Jell, H.; Fulford, J.; Carter, J.; Nyman, L.; Bailey, S.J.; Jones, A.M. Dietary nitrate supplementation improves sprint and high-intensity intermittent running performance. Nitric Oxide Biol. Chem. 2016, 61, 55-61. [CrossRef] [PubMed]

42. Lopez-Samanes, A.; Gomez Parra, A.; Moreno-Perez, V.; Courel-Ibanez, J. Does Acute Beetroot Juice Supplementation Improve Neuromuscular Performance and Match Activity in Young Basketball Players? A Randomized, Placebo-Controlled Study. Nutrients 2020, 12, 188. [CrossRef] [PubMed]

43. Marechal, G.; Gailly, P. Effects of nitric oxide on the contraction of skeletal muscle. Cell. Mol. Life Sci. CMLS 1999, 55, 1088-1102. [CrossRef] [PubMed]

44. Terraza-Rebollo, M.; Baiget, E. Effects of Post-Activation Potentiation on Tennis Serve Velocity and Accuracy. Int. J. Sports Physiol. Perform. 2019, 1-20. [CrossRef]

45. Brink-Elfegoun, T.; Ratel, S.; Lepretre, P.M.; Metz, L.; Ennequin, G.; Dore, E.; Martin, V.; Bishop, D.; Aubineau, N.; Lescuyer, J.F.; et al. Effects of sports drinks on the maintenance of physical performance during 3 tennis matches: A randomized controlled study. J. Int. Soc. Sports Nutr. 2014, 11, 46. [CrossRef]

46. Cuenca, E.; Jodra, P.; Perez-Lopez, A.; Gonzalez-Rodriguez, L.G.; Fernandes da Silva, S.; Veiga-Herreros, P.; Dominguez, R. Effects of Beetroot Juice Supplementation on Performance and Fatigue in a 30-s All-Out Sprint Exercise: A Randomized, Double-Blind Cross-Over Study. Nutrients 2018, 10, 1222. [CrossRef]

47. Rimer, E.G.; Peterson, L.R.; Coggan, A.R.; Martin, J.C. Increase in Maximal Cycling Power With Acute Dietary Nitrate Supplementation. Int. J. Sports Physiol. Perform. 2016, 11, 715-720. [CrossRef]

48. Jodra, P.; Dominguez, R.; Sanchez-Oliver, A.J.; Veiga-Herreros, P.; Bailey, S.J. Effect of Beetroot Juice Supplementation on Mood, Perceived Exertion and Performance During a 30 s Wingate Test. Int. J. Sports Physiol. Perform. 2019, 10, 1-20. [CrossRef]

49. Kramer, S.J.; Baur, D.A.; Spicer, M.T.; Vukovich, M.D.; Ormsbee, M.J. The effect of six days of dietary nitrate supplementation on performance in trained CrossFit athletes. J. Int. Soc. Sports Nutr. 2016, 13, 39. [CrossRef]

50. Coggan, A.R.; Leibowitz, J.L.; Kadkhodayan, A.; Thomas, D.P.; Ramamurthy, S.; Spearie, C.A.; Waller, S.; Farmer, M.; Peterson, L.R. Effect of acute dietary nitrate intake on maximal knee extensor speed and power in healthy men and women. Nitric Oxide Biol. Chem. 2015, 48, 16-21. [CrossRef]

51. Coggan, A.R.; Peterson, L.R. Dietary Nitrate and Skeletal Muscle Contractile Function in Heart Failure. Curr. Heart Fail. Rep. 2016, 13, 158-165. [CrossRef] [PubMed]

52. Kovacs, M.S. Movement for Tennis: The Importance of Lateral Training. Strength Cond. J. 2009, 31, 77-85. [CrossRef]

53. Martin, K.; Smee, D.; Thompson, K.G.; Rattray, B. No improvement of repeated-sprint performance with dietary nitrate. Int. J. Sports Physiol. Perform. 2014, 9, 845-850. [CrossRef] 